\title{
Il dovere di essere ospitali. Notazioni su giustizia e accoglienza
}

\author{
The duty of being hospitable. Reflections on justice and reception
}

\author{
Guido SARACENI \\ Facoltà di Giurisprudenza. Università degli Studi di Teramo \\ https://orcid.0000-0002-9734-3485 \\ gsaraceni@unite.it
}

RECIBIDO: 13/03/2019 / ACEPTADO: 31/10/2019

\begin{abstract}
Riassunto: L'epoca che stiamo vivendo potrebbe essere definita come l'epoca delle migrazioni globali. Ingenti masse di individui - nella più gran parte provenienti da Paesi tormentati dalla fame, dalle epidemie o dalle guerre - attraversano il mare, l'oceano o il deserto alla ricerca di lidi più sicuri dove trovare riparo e accoglienza. Dal canto loro, i popoli occidentali avvertono la pressione di questo massiccio esodo, ne ponderano con crescente preoccupazione le conseguenze - in termini economici e di ordine pubblico -, si dividono tra i pochi che vorrebbero garantire ai migranti ospitalità e accoglienza e i molti che preferirebbero alzare mura sempre più alte per segregare la miseria e la disperazione dall'altra parte di una frontiera. Da dove nasce questa diffidenza nei confronti dello straniero? Quali sono i meccanismi psicologici e sociologici che ne favoriscono la diffusione? Prendendo spunto da simili domande, il presente saggio intende rintracciare nel dovere di ospitalità la posizione originaria e fondativa della filosofia del diritto.
\end{abstract}

Parole chiave: stranieri; ospitalità; diritti umani; giustizia.

\begin{abstract}
The era in which we are living could be defined as the era of global migration. Masses of inividuals - in the most part coming from countries tormented by hunger, epidemics or wars - are crossing the sea, the ocean or the desert, in search of safer shores where to find shelter and hospitality. From their part, Western people feel the strong pressure of this massive exodus, evaluate with increasing concern the consequences - for economy and public order -, and basically divide themselves into two different groups: the few who want to offer hospitality and the many who would rather raise higher and higher walls to segregate misery and despair on the other side of a frontier. Where does the distrust of the foreigner come from? What are the psychological and sociological mechanisms that favor its diffusion? Could we find a philosophical guideline to address such problems? Inspired by such questions, the present essay detect in the duty of hospitality the original and foundational position of philosophy of law.
\end{abstract}

Keywords: strangers; hospitality; human rights; justice.

Sumario: 1. QUESTIONI PRELIMINARI, LINGUISTICHE E PSICANALITICHE. 2. LOSTRANIERO È POVERO. 3. LINEAMENTI FILOSOFICI: LA DIMORA E IL VOLTO. 4. IL DOVERE DI ESSERE OSPITALI.

\section{QUESTIONI PRELIMINARI, LINGUISTICHE E PSICANALITICHE}

L

a ricerca etimologica ci consente di sostenere che il termine «hospes» deriva da «un antico composto» in cui è possibile rintracciare la presenza del «membro pet- in alternanza con pot- che significa 'signore' [...]». Originariamente, esso indicava colui il quale offriva accoglienza, significando, letteralmente, «signore dell'ospite». Dalla medesima radice sarebbe derivato successivamente il termine «straniero» - non però nel senso della generica appartenenza ad una diversa comunità politica, quanto nel senso di un estraneo la cui identità 
è riconosciuta e rispettata. Questo identico seme linguistico avrebbe generato anche il termine hostis che, ci informa Cicerone, possedeva originariamente un significato ambiguo e polisemico, tanto da poter essere utilizzato per indicare, indifferentemente, il nemico l'ospite e lo straniero ${ }^{1}$.

Ma quando si diffusero i termini «peregrinus» ed «hospes» per indicare, rispettivamente, lo «straniero» e l'«ospite innocuo», al lemma «hostis» fu riconosciuto un valore eminentemente negativo. A partire da quel momento, questa parola entrò a far parte del vocabolario dell'antagonismo, risultando semanticamente contrapposta al termine «cives» che serviva per indicare chi, essendo cittadino era anche civile - in ragione della sua raffinatezza culturale -, ma non suscitava interesse e non destava preoccupazione, perché già iscritto all'interno di logiche parentali ed amicali. Insomma, il cives non pone alcun problema - rappresentando la tessera di un mosaico etnico e culturale sostanzialmente omogeneo: egli è rassicurante perché in-genuus -, mentre «hostis» è il termine che indica il nemico pubblico e straniero, «inimicus» fa riferimento al nemico privato ed interno e «competitor» viene utilizzato per denotare l'avversario in un conflitto di interessi.

La ricchezza semantica sin qui brevemente illustrata era sicuramente nota a Sigmund Freud, quando, nel marzo del 1919, iniziò a scrivere $A l$ di là del principio di piacere. La stesura di questo volume, destinato a diventare una pietra miliare nella letteratura psicoanalitica mondiale, durò per ben quindici mesi. Leggendo una lettera inviata da Freud allo psichiatra ungherese Sándor Ferenczi e datata 12 maggio 1919, apprendiamo che simili tempi di gestazione - davvero insoliti per un uno studioso prolifico come Freud - non furono dovuti tanto alla complessità dei temi trattati, quanto al fatto che l'Autore si bloccò subito dopo aver scritto le prime pagine, decidendo di rimandare alle vacanze estive il completamento dell'opera: sentiva l'urgenza di tornare su di un tema solo accennato, circa un decennio prima, in una breve nota di Totem e tabü ${ }^{2}$.

1 Con riguardo all'origine etimologica del termine, BENVENISTE, E., Vocabolario delle istituzioni indoeuropee. Economia, parentela, società, Einaudi, Torino, 2001; CURI, U., Straniero, Raffaello Cortina, Milano, 2010, pp. 68-69.; VITALE, E., Ius migrandi, figure di erranti al di qual della cosmopoli, Bollati Boringhieri, Torino, 2004, pp. 33 e ss; GENTILI, D., «Topografie politiche. Spazio urbano, cittadinanza, confini», in Walter Benjamin e facques Derrida, Quodlibet, Macerata, 2009; pp. 62-64.

2 Un riferimento alla corrispondenza epistolare citata nel testo si trova in FreUd, S., Opere, Parte II, Bollati Boringhieri, Torino, 2006, p. 759. 
Il manoscritto che costrinse Freud ad interrompere la stesura del suo capolavoro, ed al quale egli lavorò alacremente tra il maggio ed il luglio del 1919, si chiama Das Unheimliche ${ }^{3}$ - titolo tradotto più o meno felicemente in italiano con «Il perturbante». In questo saggio il Padre della psicanalisi indaga l'etimologia del termine Unheimlich - «esplorando il significato che l'evoluzione della lingua ha sedimentato nel termine»-, prefiggendosi inoltre di «collazionare ciò che, riferito a persone e a cose, a impressioni sensoriali, a esperienze e situazioni, evoca in noi il senso del perturbante» al fine di sceverare con precisione in cosa consista, esattamente, la peculiare essenza angosciosa di «ciò che perturba» ${ }^{4}$.

Freud annota in quel suo scritto che la parola Unheimlich sembra rappresentare l'antitesi del termine Heimlich, che significa «familiare». Da ciò sarebbe ovvio dedurre che qualcosa suscita spavento proprio perché non è familiare. Tuttavia, non ogni novità risulta, per ciò solo, spaventosa, «bisogna aggiungere qualcosa all'inconsueto perché diventi perturbante $»^{5}$. Inoltre, l'Autore giunge a concludere che il termine Unbeimlich sarebbe in realtà «meno oppositivo» di quanto non sembrerebbe indicare la presenza del prefisso «un»; più esattamente, dovrebbe essere considerato come una variante del suo contrario, il termine Heimlich ${ }^{6}$.

3 Das Unheimliche è stato pubblicato per la prima volta su «Imago», vol. 5, 1919, pp. 297-324, per essere poi pubblicato almeno altre tre volte in altrettante edizioni freudiane. Il saggio di cui mi avvalgo in questo scritto è stato tradotto da Silvano Daniele ed è incluso nella esaustiva antologia Opere, cit., pp. 760-801. Per un commento de Il perturbante, si veda RESTA, C., L'Estraneo. Ostilità e ospitalità nel pensiero del Novecento, Il Nuovo Melangolo, Genova, 2008, pp. 51-63; Esposito, R., Communitas. Origine e destino della comunità, Einaudi, Torino, 2006, pp. 20-25; CURI, U, Straniero, cit., pp. 21-55; CAROTENUTO, A., Il fascino discreto dell'orrore, Bompiani, Bologna 2002, pp. 43-56.

4 Opere, cit., pp. $760-761$.

5 Ivi, p. 761.

6 Non è forse privo di significato che Freud inizi la sua analisi dal lemma Heimlich affermando che esso «non è univoco, ma appartiene a due cerchie di rappresentazioni che, senza essere antitetiche, sono tuttavia parecchio estranee l'una all'altra: quella della familiarità, dell'agio e quella del nascondere, del tener celato» per questo motivo, «si tratta di un termine che sviluppa il suo significato in senso ambivalente, fino a coincidere in conclusione con il suo contrario: unheimlich» (Il perturbante, cit., pp. 765-767). Peraltro, Freud era convinto che il linguaggio dell'inconscio fosse paragonabile al linguaggio arcaico - d'altronde, egli aveva già recensito, nel 1910, un importante testo di Abel sui simboli primari - perché entrambi non conoscono negazione o principio di contraddizione; tuttavia, questa teoria fu aspramente criticata da Benveniste, ad avviso di quest'ultimo autore, non può esistere un linguaggio che non rispetti il principio di non-contraddizione. Dobbiamo comunque rilevare che l'ambito epistemologico a cui appartengono le tesi appena citate è profondamente diverso: è del tutto evidente che quando Freud parla di un linguaggio della psiche non intende il termine «linguaggio» nello stesso identico modo in cui lo potrebbe intendere un filologo. Sulla querelle da ultimo accennata, si veda RESTA, C., L'Estraneo, cit., p. 50. 
Al fine di suffragare una tesi che prende corpo sin da queste primissime argomentazioni, Freud analizza due opere letterarie: la prima è un racconto di Hoffmann datato 1816 ed intitolato Der Sandman ${ }^{7}$, la seconda è Gli elisir del diavolo - sempre di Hoffmann - e narra lo scontro tra due personaggi, l'uno il doppio dell'altro ${ }^{8}$. Notiamo come Freud usi alternare esempi tratti dalla narrativa - dal Faust a Mark Twain - a resoconti di esperienze vissute in prima persona, alla disamina di veri e propri casi clinici; solo alla fine del saggio egli opera una netta distinzione tra poesia e vita reale, affermando che sperimentiamo «direttamente» una sensazione perturbante «quando complessi infantili rimossi sono richiamati in vita da un'impressione o quando convinzioni primitive superate sembrano aver trovato una nuova convalida» ${ }^{9}$. Possiamo quindi concludere che per Freud il sentimento del perturbante non nasce da qualcosa di estraneo al vissuto del soggetto, ma proviene dalle più recondite profondità della mente: si tratta di un vissuto «che avrebbe dovuto rimanere nascosto e che è invece affiorato» ${ }^{10}$.

Per questo motivo, molti e autorevoli studiosi hanno creduto di poter individuare nello straniero «una figura del perturbante ${ }^{11}$. Nelle sue linee essenziali, si tratta di uno spunto parecchio interessante e condivisibile. Eppure,

7 Si tratta della storia di un uomo (Nathaniel) che nella sua infanzia aveva identificato «l'uomo della sabbia» - una sorta di uomo nero di cui gli parlava la governante - con un amico di famiglia, l'avvocato Coppelius. Durante gli studi universitari, Nathaniel si innamora di Olimpia, una bambola creata da Lazzaro Spallanzani che il protagonista spia osservandola grazie ad un binocolo. Quando finalmente Nathaniel riesce a liberarsi da questa insana ossessione, mentre, sul punto di riconciliarsi con la sua antica amata, contempla dall'alto di una torre la città sottostante, incrocia lo sguardo di Coppelius che, ammaliandolo, lo spinge a gettarsi nel vuoto.

8 Per quel che concerne il valore simbolico dello specchio, interpretato in chiave psicoanalitica e letteraria, si veda CARotenuto, A., Il fascino discreto dell'orrore, Cap II «Specchio delle mie brame», cit., p. 27 e ss.

9 Il perturbante, cit.,

10 CURI, U., Straniero, cit., p. 49

11 Commentando questa teoria, Umberto Curi annota: «la domus, il rassicurante ambito dei nostri affetti - il luogo nel quale ci ritiriamo per sentirci protetti, nel quale crediamo di poter trovare accoglienza e comprensione -, ospita, in realtà anche ciò che credevamo essere esterno e remoto rispetto ad essa» (Straniero, cit., p. 53). In maniera sostanzialmente coerente e parecchio significativa, Roberto Esposito sostiene che la rimozione primigenia che genera il sentimento del perturbante sarebbe da identificare con l'uccisione del padre. Quest'ultimo autore afferma che gli individui siglano il contratto sociale, rinunciando al proprio potere ed alla propria libertà, non per la paura della morte - come sarebbe sin troppo facile inferire dalla lettura dei testi hobbesiani a cui egli opera costante e preciso riferimento -, ma per la paura di un morto, «o meglio, del suo ritorno come morte per tutti coloro $-\mathrm{i}$ fratelli - che l'hanno una prima volta data e così per sempre fatta propria» (Communitas, cit., p. 22). 
corre l'obbligo di operare alcune precisazioni preliminari: la prima, e forse la più importante, è che in questo scritto lo straniero non viene mai citato esplicitamente come un esempio di ciò che è perturbante. Quando il Padre della psicanalisi propone la sua tassonomia esplicativa, egli cita a più riprese la paura di essere evirati; il buio; la solitudine; la scoperta che il nostro pensiero può realmente influenzare gli eventi; le coincidenze; la perdita dell'orientamento che ci induce a girovagare senza meta, tornando più volte al punto di partenza; gli arti mozzati; l'organo genitale femminile ed il sospetto che un oggetto possieda vita propria. Si tratta di una lista parecchio esaustiva e dettagliata. Per questo motivo, credo che qualsiasi riflessione che intenda criticare il carattere perturbante dello straniero, pur innestandosi legittimamente nell'impianto generale delle riflessioni freudiane, debba dichiarare esplicitamente che si sta spingendo oltre la lettera del testo, prospettandone un significato forse plausibile, ma certamente ulteriore.

La seconda importante precisazione è che, a mio avviso, ad essere freudianamente perturbante, non è, propriamente, lo straniero, ma l'estraneo; non solo e non tanto perché all'interno del racconto di Hoffman che tanto ha affascinato Freud colui il quale turba il piccolo protagonista sino a determinarne - molti anni più tardi - la tragica morte per suicidio non è affatto uno straniero, ma soprattutto perché l'irruzione del volto dell'altro è un'esperienza ben più radicale della successiva opposizione - di matrice chiaramente giuridica o forse più propriamente «politica» - tra cittadino e straniero. Molto prima di entrare in qualsiasi altra forma relazionale - come, ad esempio, quella tra amico e nemico - dobbiamo saper fare i conti con l'apparire di figure «non genitoriali» nei confronti delle quali tendiamo ad avere un atteggiamento ambiguo, genuinamente sospeso sul confine tra paura e curiosità ${ }^{12}$.

Come è stato correttamente osservato: nell'istante stesso «in cui emerge il Due (e la radice di $d \bar{y} o$ è la stessa del verbo timore, deìdo, e del termine che indica il tremendo, lo spaesante, deinòs)», emerge anche la meraviglia che «inquieta e spaventa», stimolando «la ricerca intorno alla sua origine, alle sue

12 Sin dai primi anni di età, quando ancora non sanno nulla di stati e nazioni, i bambini hanno un atteggiamento ambiguo nei confronti di chi non appartiene al nucleo familiare, nascondendosi dietro le gambe dei genitori per sbirciare le fattezze del soggetto estraneo dal quale risultano affascinati ed impauriti ad un tempo; in tal modo, inscenano un rituale di avvicinamento che esprime, in maniera davvero lapalissiana, quel rapporto di attrazione/repulsione ci lega alla figura dell'altro, ed alla sua sconvolgente epifania (TABBONI, S., Lo straniero e l'altro, Liguori, Napoli, 2006, p. 11). 
interne relazioni, al suo stesso fine» ${ }^{13}$. Detto con altre parole, lo spaesamento causato dal rapporto con Altri dipende essenzialmente dal fatto che l'a-venire altrui non è prevedibile, si tratta di un evento che - destituendo ogni costituzione di soggettività - rappresenta l'invenzione dell'impossibile ${ }^{14}$. Se l'estraneo è, per sua natura, inquietante, a maggior ragione risulterà inquietante lo straniero, con il suo bagaglio di provocazioni, di malattie e di pretese.

\section{LO STRANIERO È POVERO}

L'analisi sociologica sembra confermare la bontà di questa profonda intuizione freudiana. Georg Simmel ha infatti spiegato che tutte le relazioni sociali sono, in una qualche misura, «ambivalenti»: accettiamo di parteciparvi solo a patto di poter conservare una certa distanza dai vincoli stabiliti tramite di esse. Prendiamo volontariamente parte a rapporti fatti di obblighi e diritti reciproci, ma sentiamo il bisogno di lasciare sempre aperta una via di fuga, di conservare uno spazio di libertà. Ciò sta a significare che - prescindendo da particolari esperienze di amore e di ascesi mistica - l'essere umano tende a rifiutare le relazioni totalizzanti, preferendo preservare sempre e comunque una propria zona di autonomia rispetto al ruolo che viene ad assumere all'interno di un determinato contesto. Questo è il tema che l'Autore sviluppa nel celebre Exkurs über den Fremden in cui lo straniero, il povero ed il reietto vengono presi ad esempio come figure paradigmatiche dell'ambivalenza. Tutti e tre esistono all'interno di un gruppo in ragione del loro esserne esclusi ${ }^{15}$. Non è un caso se l'Exkurs fa parte di in un più ampio capitolo dedicato a $L o$ spazio e gli ordinamenti spaziali della società. L'analisi sociologica simmeliana

13 Prosegue CACCIARI, M., «interrogarsi sul differire comporta interrogarsi sull'identità, meravigliarsi del molteplice (che l'ente sia molteplice) inizia al 'ricordo' dell'Uno. Non basterà conoscere i distinti, 'analizzarli', è necessario chiedersi come la scissione sia avvenuta, quale logos l'abbia prodotta», poiché «dalla meraviglia per l'emergere e lo stare dei Due [...] s'impone, filosoficamente-politicamente, il problema della relazione tra uno e molti» (Geofilosofia dell'Europa, Adelphi, Milano, 1994, pp. 12-13).

14 Resta, C., L'evento dell'altro: etica e politica in facques Derrida, Torino, 2003, in particolare, si veda il Capitolo I, $\$ 3$, L'invenzione dell'altro, p. 24 ss.

15 «Lo straniero è un elemento del gruppo stesso, non diversamente dai poveri e dai molteplici 'nemici interni', un elemento la cui posizione immanente e di membro implica contemporaneamente un di fuori e un di fronte» (Sociologia, Meltemi, Torino, 1998, 580). Per una disamina dell' Excurs, TABвoni, S., Lo Straniero e l'altro, cit., p. 40 ss; Perrone, L., Da straniero a clandestino. Lo straniero nel pensiero sociologico occidentale, Liguori, Napoli, 2005, p. 46 ss. 
ci ricorda infatti come ciascun gruppo sociale tenda a collocare se stesso al centro dell'universo, relegando tutto il resto in periferia. La posizione sociologica occupata dallo straniero può essere quindi compresa solo attraverso le coordinate cartesiane del dinamismo e della immobilità. Lo straniero, grazie al suo arrivo, avvicina ciò che è lontano, innescando, in tal modo, una polarità dialettica nei confronti della staticità - della fissità - in cui si trova la società che lo ospita ${ }^{16}$.

A maggior riprova delle considerazioni sin qui sviluppate, molte e diverse culture hanno elaborato un giudizio ambiguo nei confronti degli stranieri, spesso considerati, incivili, sporchi, infantili, ed al tempo stesso dotati di un fascino ancestrale proprio perché ancora non civilizzati ${ }^{17}$. Erodoto e Plinio il Vecchio descrivevamo i popoli lontani come se appartenessero ad una specie diversa di uomini, caratterizzata da usanze incivili, capacità straordinarie e da corpi aberranti e mostruosi ${ }^{18}$; parimenti, i racconti dei gran tour continentali ed i resoconti sulle Indie insistevano sulla affascinante sensualità delle incivili popolazioni straniere ${ }^{19}$.

Se volessimo tuttavia provare a dire qualcosa di ulteriore e di altrettanto sensato circa l'essenza dello straniero, potremmo sostenere che egli è, per definizione, «povero». Questa tesi trova un primo elemento di conforto nel legame etimologico che unisce la parola tedesca Elend («miseria») all'antico sassone «eli-lendi» («altra terra», «terra straniera») ${ }^{20}$; è comprovata sociologicamente dalle tesi simmeliane che, come abbiamo avuto modo di ricordare,

16 Per quanto lo straniero possa contribuire alla vita sociale, per quanto egli possa essere «immerso» all'interno del gruppo, si troverà sempre e comunque «in una posizione di contrapposizione rispetto a quella della totalità: dando e ricevendo, essendo trattato bene o male da questa, legato a questa da sentimenti di appartenenza o di esclusione» (Sociologia, Torino, 1997, p. 170).

17 Su questa ambivalenza insiste TABBONI, S., Lo Straniero e l'altro, cit., pp. 7-35.

18 Erodoto descrive gli Egizi dicendo che mangiano all'aperto e fanno i bisogni in casa; che le donne orinano in piedi e gli uomini accucciati; che le donne vanno al mercato e gli uomini restano in casa a tessere; gli Arimaspi hanno un unico occhio in mezzo alla fronte; gli Ataranti, popoli della Libia, non hanno un nome proprio; gli Ausei non hanno famiglia, la natura del barbaro è mostruosa, si tratta di «una specie differente di uomini: essi non vengono da un'altra città» (CURI, U., Straniero, cit., p. 79).

19 In definita, è stato correttamente osservato che l'ambiguo ruolo sociale degli stranieri inquieta ed affascina i cittadini, mettendone sotto scacco la razionalità binaria, inficiandone la sicurezza, condannando a sicura sconfitta «ogni possibile linguaggio dell'unicità» (CURI, U., Straniero, cit., p. 13).

20 Sul punto, Conte, A. G., «Elend: il linguaggio dell'alterità», in AA. VV. (R. Astorri e F. A. Cappelletti [a cura di]), Lo Straniero e l'ospite. Diritto Società Cultura, Giappichelli, Torino 2002, pp. 6 ss. 
assimilano la posizione sociologica in cui si trovano lo straniero ed l'indigente; trova conforto nelle riflessioni di uno dei più importanti filosofi del novecento ed una giustificazione teologica nella esegesi del testo biblico che lega «il rivelarsi di Dio al gemito dello straniero», accomunando la condizione in cui si trovano il viandante ed il povero ${ }^{21}$.

Tuttavia, quando affermo che lo straniero è povero - seguendo la traccia aperta dalle suggestioni poc'anzi illustrate - non intendo in alcun modo fare riferimento ad una precarietà economica: è del tutto evidente che possono esistere stranieri che non si trovano in condizioni di bisogno, avendo a disposizione un patrimonio assimilabile, se non addirittura superiore, a quello posseduto dalla media dei cittadini. Ma lo straniero è strutturalmente povero, a prescindere da quale sia il suo reddito, perché si trova a vivere una condizione esistenziale di costitutiva indigenza che dipende da ciò che egli è , non da ciò che egli ha o non $b a$. Ovvero dalla qualità e dalla quantità delle cose che ogni straniero, in quanto tale, ignora ${ }^{22}$.

Per avere una riprova del fatto che l'essere straniero dipende essenzialmente da una difettività culturale, basti pensare che, in greco, la parola «barbaro» possedeva un referente eminentemente linguistico, attribuendo il massimo e più dispregiativo grado dell'estraneità a colui il quale, non riuscendo ad esprimersi fluentemente nell'idioma attico, balbettava; questo stesso significato sarebbe da attribuire alla parola latina balbus (da cui, appunto, il termine italiano «balbuziente»); allo stesso modo, gli slavi d'Europa chiamavano il loro vicino tedesco nemec, «il muto»; i maya dello Yucatan definivano nunob («i muti») gli invasori toltechi, mente i maya cackchiquel si riferivano ai maya

21 Di SANTE, C., Lo straniero nella bibbia. Saggio sull'ospitalità, San Paolo, Troina (EN), 2002, pp. 28 31.

22 Mi sembra che possano essere interpretate in questo modo le parole di Hans Jonas laddove, commentando la letteratura gnostica mandea, il filosofo tedesco scrive che il destino dello Straniero è di essere «solitario, senza protezione, incompreso e incapace di comprendere, in una situazione piena di pericoli [...] Egli che non conosce le strade del nuovo paese girovaga sperduto; se impara a conoscerle troppo bene dimentica di essere uno straniero e si perde in un senso inverso, soccombendo alle attrattive del mondo straniero e diventando estraneo alla sua propria origine» (Lo gnosticismo, SEI, Torino, 1991, p. 69). Con ciò non intendo in alcun modo negare che «l'altro si rivela realmente come altro in tutta l'intensità della sua esteriorità, quando irrompe come il non abituale o quotidiano, come lo straordinario [...] come il povero, l'oppresso, colui che al margine della strada, fuori del sistema, mostra il suo volto sofferente che lancia tuttavia una sfida» (Dussel, E., Filosofia della liberazione, Queriniana, Brescia, 1992, p. 108). Intendo tuttavia sostenere che questo essere «al margine della strada» non debba essere interpretato in termini esclusivamente economici. 
mam come ai «balbuzienti» ${ }^{23}$. Un uomo è dunque straniero quando non conosce la lingua e dunque ignora la religione, il cibo e più in generale «i tempi» ed $\mathrm{i}$ «luoghi» della terra in cui si trova ${ }^{24}$. D'altronde, è solo interpretando in questo modo la condizione dello straniero che diviene possibile comprendere come si possa essere stranieri all'interno dello Stato in cui si è nati ed in cui si vive - esattamente come Socrate che, a fronte dei suoi giudici, dichiarò di essere estraneo all'oratoria, alla retorica e più in generale alle tecniche del diritto e di sentirsi dunque spaesato, «semplicemente uno straniero senza capacità, senza sostegni e senza risorse. Come se nella realtà fossi un uomo di un altro paese $\gg^{25}$.

Fermo restando che essere stranieri significa trovarsi in una condizione di manifesta inferiorità, non è dunque facile comprendere dove nasca il diffuso senso di ostilità che, in questi ultimi anni, ha determinato la creazione di barriere atte ad escludere e discriminare i più deboli tra gli esseri umani, evitando la commistione - o finanche il confronto - con coloro i quali vengono considerati «ontologicamente» diversi ${ }^{26}$. Rimarcando la profondità di questa inquietudine, uno dei più noti antropologi del Novecento ha giustamente notato che i testi sacri enfatizzano con tanta decisione la comune appartenenza

23 Todorov, T., La conquista dell'America e il problema dell'altro, Einaudi, Torino, 2014, p. 92.

24 «Se invece parlasse già la nostra lingua, con tutto ciò che la cosa implica, se condividessimo già tutto ciò che insieme alla lingua si condivide, lo straniero sarebbe ancora uno straniero e potremmo parlare di ospitalità?» (DERrIDA, J., Sull'ospitalità, Raffaello Cortina, Milano, 2000, p. 46). Anche per questo motivo, «Lo straniero non è portatore di una differenza oggettiva, non è pensabile come elemento o soggetto indipendente; dal punto di vista sociologico lo straniero non esiste mentre esiste la relazione noi/altro», Perrone, L., Da straniero a clandestino, cit., p. 46.

25 Per un commento, BilotTa, B.M., «L'ospite poco ospite», in B. M. Bilotta e F. A Cappelletti (a cura di), Il diritto d'asilo, Cedam, Padova, 2006, pp. 193 e ss.

26 «Che cosa ci spaventa e ci inquieta in quei volti che vediamo ogni giorno assieparsi infreddoliti e diffidenti, talvolta spavaldi, o pieni di speranza, ai parapetti delle navi, negli atri delle stazioni? Sono fuggiti da case povere e bruciate. Altre case povere li attendono, caserme squallide, carceri. Ci spaventa forse proprio il loro non avere nulla, la loro tenacia di profughi, di esiliati, che li sostiene e li rende capaci di sopportare viaggi allucinanti, antica e forte quanto la fame» (TARTER, S., evento e ospitalità. Levinas, Derrida e la questione straniera, Cittadella, Assisi, 2004, pp. 101-102). «La barriera che la nostra società erige contro di oro non è costituita soltanto dalle forme empiriche di violenza e di discriminazione [...] ma è in primo luogo politica. Come tale non dispone soltanto delle consuete forme di difesa dei confini (l'apparato repressivo civile e militare), ma di una simbologia che trasforma la distinzione puramente empirica tra noi e loro in una contrapposizione ontologica, cioè tra mondi radicalmente opposti» (DAL LaGo, A., Non-persone. L'esclusione dei migranti in una società globale, Feltrinelli, Milano, 2004, p. 43). 
di tutte le razze ad una stessa famiglia proprio in ragione della fortissima difficoltà umana ad avvicinarsi a chi è diverso, stabilendo un rapporto con altre tradizioni culturali ${ }^{27}$. Molto probabilmente, si tratta del fatto che noi abbiamo paura di riconoscerci nel volto dello straniero e di capire che ne condividiamo il destino. I cittadini temono che un qualsiasi contatto con lo straniero possa determinare un contagio, perché - attraverso il dialogo - la povertà del nuovo arrivato potrebbe dilagare all'interno del corpo sociale, trasformandosi subitaneamente nella povertà di tuttii ${ }^{28}$.

Il punto è che l'arrivo di uno straniero, mette in questione le nostre certezze, provocando le tradizionali categorie concettuali, mettendone alla prova la purezza e la fondatezza ${ }^{29}$; ciò accade non solo perché gli stranieri sono latori di abitudini, leggi e religioni alternative e spesso conflittuali con le nostre, ma anche e soprattutto perché essi revocano in dubbio la precisione delle coordinate cartesiane a partire dalle quali abbiamo pensato e strutturato il nostro mondo: accostarsi ad uno straniero significa accettare che la nostra lingua non è $l a$ lingua, la nostra religione non è $l a$ religione e la nostra cultura non è $l a$ cultura; accostarsi ad uno straniero significa toccare con mano la contingente relatività delle nostre leggi, delle nostre istituzioni, del mondo che abbiamo costruito. Insomma, significa sperimentare la nostra creaturale indigenza e scoprire che la società in cui viviamo non è assoluta e quindi non è perfetta, perché non è unica.

La contagiosa malattia della povertà è dunque il vero dono che lo straniero porta con sé. Grazie al contatto con lo straniero noi comprendiamo di avere un confine e siamo paradossalmente messi nella condizione di divenire realmente proprietari del nostro terreno.

27 STRAUSS, C. L. Razza e storia e altri studi antropologici, Einaudi, Torino, 1967; per un commento, TABBONI, S., Lo Straniero e l'altro, cit., p. 16 e ss.

28 In tal modo, propongo una interpretazione alternativa e speculare rispetto all'idea che la conflittualità tra $i$ cittadini e gli stranieri nasca da una fascinazione, da un inappagabile desiderio di appropriazione, ovvero dal fatto che «ci si accorge che l'altro possiede qualcosa che noi non possediamo e che vorremmo possedere» (BILOTTA, B. M., L'ospite poco ospite, cit., p. 182). Con ciò, non intendo affatto negare la correttezza dell'analisi girardiana riproposta da Bilotta, ma ipotizzare una diversa radice per il disagio causato dalla presenza di uno straniero.

29 Per questo motivo, «lo straniero si fa personificazione di un'interrogazione, ma anche di un interrogatorio, che il nostro stare-insieme-nel-mondo rivolge a se stesso. Grazie allo straniero esso non può fare in meno di interrogarsi sulla propria cultura, sulla propria teologia, sulla propria filosofia, sull'etica e sulla politica, sulla lingua e sulle istituzioni giuridiche in vigore, in definitiva sul modo con cui mette in pratica l'ospitalità» (CocColinI, G., Ospite Ostaggio Nemico, Pardes, Bologna, 2004, p. 11). 
Le riflessioni sin qui sviluppate con riguardo al tema dello straniero trovano un banco di prova estremamente interessante nel pensiero di uno tra $\mathrm{i}$ massimi pensatori del XX secolo: Emmanuel Lévinas.

\section{LINEAMENTI FILOSOFICI: LA DIMORA E IL VOLTO}

«L'ontologia è davvero fondamentale?» ${ }^{30}$. La speculazione filosofica di Lévinas prende le mosse da questa domanda originaria, configurandosi, nella sua interezza, come il tentativo di fornire una risposta ad un interrogativo tanto radicale quanto urticante. Proponendo «una via d'uscita dall'essere» e provando a superare i grandi maestri novecenteschi che l'avevano preceduto, Lévinas propone un'interpretazione personale della fenomenologia husserliana e dell'ontologia heideggeriana, rimarcandone i rispettivi limiti ed utilizzandone in maniera sicuramente innovativa, ed in un certo qual modo «spuria», i concetti fondativi ${ }^{31}$.

Nel contesto culturale successivo alla II Guerra, essenzialmente incentrato sulla natura sociale dell'essere umano, l'Autore pubblica l'agile, ma densissimo, Le Temps et l'Autre. In tal modo prende esplicitamente le distanze dall'ontologia parmenidea, sostenendo - attraverso l'anticipazione di molti temi che saranno ripresi e sviluppati in Totalità e Infinito - che la relazionalità e il tempo costituiscono la «struttura ultima» dell'essere. Il centro di gravità di Le Temps et l'Autre è rappresentato dal sentimento dell'orrore - uno Stimmung

30 LÉVINAS, E., «L'ontologia è fondamentale?», in Tra di noi. Saggi sul pensare-all'altro, Jaca Book, Milano, 2000, 29-40.

31 Per dirla con le sferzanti parole di JACQUES DERRIDA, «Si potrebbe certamente mostrare come Lévinas scomodamente installato - e già per la storia del suo pensiero - nella differenza tra Husserl e Heidegger, critichi sempre l'uno dei due con uno stile e secondo uno degli schemi desunti dall'altro, finendo col rimandarli insieme dietro le quinte come compari nel «gioco dello Stesso» e complici nello stesso colpo di forza storico filosofico» («Violenza e metafisica», in Scrittura e differenza, Einaudi, Torino 2004,124). In maniera sinceramente meno urticante, FranCESCa SAlvarEZZA nota che «Lévinas, da un lato, legge Husserl con e alla luce di Heidegger ma contro Heidegger stesso, dall'altra legge Heidegger alla luce di Husserl, fino a colpire entrambi nel segno di una vibrata critica all'imperialismo del logos», più specificamente, egli «da un lato, ricorre a Heidegger per correggere il residuo teorecistico che legge in Husserl, dall'altro lato si serve della heideggeriana nozione di essere per imprimervi un movimento che è di tipo fenomenologico, nella misura in cui entra in gioco la questione del soggetto, secondo un'accezione non logica ma fattuale ed esistenziale» (Emmanuel Lévinas, Milano, 2003, p. 11 e 24). 
che Lévinas contrappone esplicitamente alla angoscia heideggeriana - declinandone le caratteristiche attraverso il riferimento alla notte, in quanto luogo di insorgenza di quella insonnia che mette sotto scacco il soggetto, dis-astrando, dis-orientando e dis-facendo il mondo ${ }^{32}$. Eppure, è proprio in questo momento di oscurità orrifica che l'individuo viene messo nelle condizioni di sperimentare un'eccessività, comprendendo finalmente che «si è tenuti all'essere, tenuti a essere $>^{33}$.

Nel tentativo di restare il più possibile fedele alla fenomenologia husserliana, piegando tuttavia il pensiero fenomenologico verso una direzione sconosciuta allo stesso Husserl, Lévinas denuncia quindi l'impossibilità del nulla, avendo cura di specificare che - sebbene il nulla non sia un vero e proprio oggetto della conoscenza - siamo tuttavia in grado di averne un'esperienza sui generis, in quanto esperienza della passività; ovvero, siamo in grado di venire a contatto con la neutralità, intesa come impossibilità radicale del porsi e, al tempo stesso, come impossibilità di farla finita con questa impossibilità ${ }^{34}$.

In maniera solo apparentemente paradossale, Lévinas prende dunque le mosse dalla considerazione che la solitudine rappresenta la condizione originaria dell'io. Più esattamente, egli considera che, in senso strettamente filosofico e dunque a prescindere da qualsivoglia riflessione sociologica o

32 «Consideriamo l'insonnia [...] L'insonnia è caratterizzata dalla coscienza che questa situazione non finirà mai, cioè che no c'è più alcun mezzo per ritrarsi dalla vigilanza alla quale si è costretti. Vigilanza senza alcuno scopo. Nel momento in cui vi si è inchiodati, è già perduta ogni consapevolezza del suo punto di partenza o del suo punto d'arrivo [...] Con una vigilanza che esclude ogni possibilità di ricorrere al sonno caratterizzeremo appunto l'il y a e il modo in cui l'esistere si afferma nel suo stesso annientamento» (Lévinas, E., Il Tempo e l'Altro, Il Nuovo Melangolo, Genova, 2005, p. 19).

33 «Arretrata rispetto alla Stimmung dell'angoscia, che svela il nulla come ciò che ha un rapporto con l'essere dell'essente, la Stimmung dell'orrore è ciò che definisce interamente il senso di questa dislocazione: a svelarsi ora è un nulla che si impone come un essere indifferente». In tal modo, «Levinas sta descrivendo una situazione senza via d'uscita, come vegliare anonimo in cui si conserva una traccia di soggetto, in cui permane un residuo di presenza soggettiva, che c'è e sussiste, ma solo nella forma di un'impotenza» (SALVAREZZA, F., Emmanuel Levinas, cit., p. 46; 51).

34 «Immaginiamo il ritorno al nulla di tutte le cose, esseri e persone. Incontreremo forse il puro nulla? Dopo questa distruzione immaginaria di tutte le cose, rimane non qualche cosa, ma il fatto che c'è [il y a]. L'assenza di tutte le cose ritorna come una presenza [...] come il mormorio del silenzio [...] L'esistere a cui stiamo tentando di accostarci è l'atto stesso di essere, che non può essere espresso con un sostantivo, che è verbo. Questo esistere non può semplicemente essere affermato, perché ciò che si afferma è sempre un essente. Ma si impone perché non lo si può negare» (LÉVINAS, E., Il Tempo e l'Altro, cit. p. 18) 
antropologica, ogni uomo è da solo ${ }^{35}$. Questa innegabile verità esistenziale possiede un aspetto positivo, nella misura in cui essa costituisce la condizione imprescindibile e prima della libertà, consentendo il dominio «virile e fiero» di una soggettività finalmente in grado di imporsi in maniera concreta sull'anonimato dell'esistenza ${ }^{36}$. Ma si rivela un'arma a doppio taglio, rischiando di rinchiudere il soggetto dietro le sbarre di una gabbia appropriativa ${ }^{37}$.

Essere soli è infatti una forma di stasi, una negazione del tempo, un perenne arenamento nelle secche del presente ${ }^{38}$ che prelude alla putrefazione: l'io ha urgente bisogno di venire salvato dalle sabbie mobili della propria coscienza, ma non può in alcun modo uscirne da solo. È necessario che sulla scena irrompa «Altri», portando in dono il futuro ${ }^{39}$.

Già Heidegger aveva articolato una penetrante analisi fenomenologica della morte, considerata come «la possibilità più propria dell'Esserci» che lo tocca nella sua stessa essenza, nel suo concreto ci, negando radicalmente ogni possibilità che lo riguarda. Tuttavia, definendo la morte come «un modo di

$35 \ll$ Ma io non sono l'Altro. Sono da solo. E' dunque l'essere in me, il fatto che esisto, il mio esistere che costituisce l'elemento assolutamente intransitivo, qualcosa ch'è senza intenzionalità, senza rapporto. Gli esseri possono scambiarsi tutto fuorché l'esistere. In questo senso, essere significa isolarsi per il fatto di esistere. Io sono monade in quanto sono» (LÉVINAS, E., Il Tempo e l'Altro, cit., pp. 15-16). Per un commento, TARTER, S., evento e ospitalità, cit., 49 ss.; FerretTI, G., La filosofia di Levinas: alterità e trascendenza, Rosenberg \& Sellier, Torino, 1996, pp. 97-108.

36 «La solitudine non è dunque soltanto disperazione ed abbandono, ma anche virilità e fierezza e sovranità. Caratteri questi che l'analisi esistenzialistica della solitudine, condotta esclusivamente in termini di disperazione, è riuscita a cancellare, facendo cadere nel dimenticanza tutti i motivi della letteratura e della psicologia romantica e byroniana che esaltano la solitudine fiera, aristocratica, geniale» (LÉVINAS, E., Il Tempo e l'Altro, cit., p. 24).

$37 \ll$ La solitudine non è tragica perché è privazione dell'altro ma perché essa è chiusa nella prigionia della propria identità, perché essa è materia [...] il tempo dato, ipostatizzato a sua volta, oggetto di esperienza, il tempo che dev' essere percorso, attraverso il quale il soggetto si trascina dietro la sua identità, è un tempo incapace di sciogliere il nodo dell'ipostasi» (LÉVINAS, E., Il Tempo e l'Altro, cit., p. 26).

38 «Come potrebbe infatti generasi il tempo in un soggetto solo? Il soggetto non può negarsi, non ha il nulla. L'alterità assoluta dell'altro istante - se il tempo non è l'illusione di una stasi - non può trovarsi nel soggetto che è definitivamente se stesso. Questa alterità può derivarmi solo da altri» (dall'esistenza all'esistente, 159-160). Introducendo Alterità e trascendenza, Pierre Hayat sottolinea come, nel pensiero di Levinas, la trascendenza non possa «essere sperimentata che come una messa in crisi della soggettività che si trova di fronte all'altro, un altro che non può contenere né assumere, e che nondimeno la mette in causa» (LÉVINAS, E., Alterità e trascendenza, Il Nuovo Melangolo, Genova, 2011, p. 11)

39 Ad avviso di Lévinas, il futuro «non può in alcun modo provenire da noi, ma solo impadronirsi di noi, cadere su di noi. Per questo il tempo non è concepibile in un soggetto solo» (FERRETTI, G., La filosofia di Levinas, cit., p. 102) 
essere che l'Esserci assume da quando c'è», Heidegger ne misconosceva la natura eventuale ${ }^{40}$. Per questo motivo, pur riconoscendo il tratto innovativo del pensiero heideggeriano e valutandone in maniera estremamente positiva il tentativo di concepire l'essere dell'uomo alla stregua di un Dasein originariamente gettato e dunque strutturalmente in balìa di qualcosa, Lévinas ne sottolinea la fallacia, rimarcando il fatto che per Heidegger l'esserci è da sempre aperto alla comprensione dell'essere ed è quindi in grado, a partire da questa comprensione, di mantenere la propria signoria su di un tempo che si compone essenzialmente di possibilità ${ }^{41}$.

Partendo dalla considerazione che il soggetto entra in contatto con il futuro solo se entra in relazione con ciò che non gli può appartenere, perché non proviene da lui, riusciamo a comprendere come la morte - o la sofferenza che ne anticipa la realizzazione - non rappresentino l'unico mistero che spezza le catene della solitudine: l'amore e la genitorialità sono altrettante, e forse più profonde, modalità della liberazione. A differenza di quanto accade con la morte, «l'amore ci invade e ci ferisce; e tuttavia l'io sopravvive in lui», ciò sta a significare che la voluttà erotica non è riconducibile a «percezione, conoscenza, possesso, fusione», ma rappresenta una forte tensione verso il «futuro puro», «ricercato nelle «carezze» con fame crescente, con promesse sempre più ricche, e in tal modo aprente la prospettiva dell'inaccessibile» ${ }^{42}$.

40 «Proprio questa passività destinata in ogni caso a mancare la presa sull'esistenza, nonostante questa possa essere com-presa» avrebbe dovuto «suggerire ad Heidegger il punto debole [...] del suo ragionamento», invece, «ciò che sfugge alla sua interpretazione è proprio il carattere di evento di questa impossibile e certissima possibilità della morte» (TARTER, S., Evento e ospitalità, cit., p. 58).

41 «La morte è, in Heidegger, evento di libertà allorchè Nel gettare luce «sul peculiare rapporto che intercorre tra l'essere e l'esserci», Heidegger si è «affrettato ad identificare il Dasein, l'esserci, in quanto articolazione dell'Essere, in quanto esser gettato nella condizione del «da» e del «ci», come immediata apertura all'Essere. In questo modo l'esserci è diventato per lui il luogo nel quale l'Essere, in quanto tempo, si apre, attraverso di lui, alla comprensione di se stesso [...] così in un primo momento, dicendo che l'esserci è fatto di tempo, Heidegger lo coglie nella sua passività, in un secondo momento, dicendo che l'esserci è da sempre aperto alla comprensione dell'essere e che a partire da questa comprensione dell'essere esso può procedere all'ordinamento degli enti, lo coglie dunque nella sua attività. L'esserci può. Di più, esso si costituisce in e attraverso questo potere, pur continuando ad essere fatto di tempo» (Lissa, G., «Dal primato della politica al primato dell'etica», in I. Kajon, E. Baccarini, F. Brezzi e J. Hansel [a cura di], Emmanuel Levinas. Prophetic Inspiration and Philosophy, Giuntina, Firenze, 2008, pp.162-163).

42 «'amore non è una possibilità, non è dovuto alla nostra iniziativa, è senza ragione, c'invade e ci ferisce e tuttavia l'io sopravvive in esso» considerata sotto questa prospettiva, la carezza erotica «è come un gioco con qualcosa che si sottrae, e un gioco assolutamente senza progetto né piano, non con ciò che può diventare nostro e identificarsi con noi, ma con qualcosa d'altro, 
Dobbiamo quindi abbandonare l'ontologia monolitica dell'essere eleatico per abbracciare una concezione dell' «esistere pluralista». Tutto ciò diviene possibile sfruttando la tensione «dialettica» che viene ad instaurarsi tra il «raggrinzimento» ipostatico dell'identità e lo schiudersi verso il futuro che solo il rapporto con «Altri» consente ${ }^{43}$.

Come premesso, queste suggestioni sono state sviluppate ed approfondite in Totalità e Infinito. In quel libro, Lévinas critica la tendenza della filosofia a rinchiudere l'essere nella luce dell'unico e totalizzante abbraccio della conoscenza; così facendo, non intende contrapporre la religione ebraico cristiana alla filosofia occidentale, ma partire dall'esperienza stessa della totalità, per contrapporre ad essa l'esperienza della trascendenza. Per raggiungere simile obiettivo, l'Autore abbandona definitivamente gli strumenti di base del metodo fenomenologico ${ }^{44}$, incardinando la propria riflessione sulle meccaniche del desiderio ${ }^{45}$. A differenza di ciò che accade con il bisogno, l'intenzionalità del desiderio non può in alcun modo essere appagata, essa «intende l'allontanamento, l'alterità e l'esteriorità dell'Altro». Di conseguenza, la relazione interpersonale non può essere interpretata sotto il segno della complementarietà: $\mathrm{i}$ termini che la costituiscono non esistono in virtù della relazione, né si risolvono al suo interno, ma vivono nella relazione - positivamente separati.

Per chiarire questa particolare concezione della soggettività può risultare utile concentrarsi sulla seconda sezione di Totalità e Infinito, ed in maniera

sempre altro, sempre inaccessibile, sempre a venire»; come diretta conseguenza la intenzionalità del piacere sessuale è «l'intenzionalità unica dell'avvenire in quanto tale»; allo stesso modo «la parternità è la relazione con un estraneo che, pur essendo altri, è me». Entrambi questi fenomeni «introducono nell'esistenza una dualità che interessa l'esistere stesso di ciascun soggetto» dato che, tramite di essa, «l'esistere stesso si reduplica» (LÉVINAS, E., Il Tempo e l'Altro, cit., pp. 52-55, corsivo mio) Per un commento, estremamente lucido si veda FerretTI, G., La filosofia di Levinas, cit., pp. 105 e ss.

43 Questa critica alla filosofia classica viene ripresa e sviluppata nel saggio Dio che viene all'idea, oggi in Alterità e trascendenza, cit., 21-45.

44 Con riguardo alla critica levinasiana della intenzionalità husserliana si veda LISSA, G., Dal primato della politica al primato dell'etica, cit., 159-161. Si consideri inoltre, SALVAREZZA, F., Emmanuel Lévinas, cit., pp. 9-35; RovatTI, P.A., Intorno a Lévinas, Unicopli, Milano 1987, p. 18 ss.

45 «La prima espressione del rapporto con l'altro, di fronte all'insufficienza degli strumenti conoscitivi, è delineata nel desiderio, che, in quanto desiderio metafisico, è essenzialmente diverso dal bisogno e quindi da ogni analisi abituale di esso; se quello infatti è movimento interno all'io e al suo mondo, nostalgia o male del ritorno, il desiderio per il filosofo è generosità che non si sazia mai, anzi, si accresce infinitamente ed è inestinguibile, perché aperto al futuro» (BREZZI, F., «Reinterrogando Levinas», in Emmanuel Levinas. Prophetic Insipiration and Philosophy, cit., 267). 
specifica sulle riflessioni dedicate dall'Autore al «godimento», inteso come «coscienza ultima di tutti i contenuti che riempiono la mia vita» ${ }^{46}$. Ciò di cui si vive, godendone, è il «fremito stesso dell'io»; non si tratta né di un oggetto di rappresentazione, che l'io è in grado di ricostruire utilizzando le proprie intenzionalità conoscitive, né di un mero «utensile alla mano», inserito nel gioco delle finalità di un ego che è stato gettato nel mondo ed è dunque progettante. Attraverso il godimento, l'io costituisce se stesso, separandosi dall'anonimo. Perché ciò sia possibile, è però necessario che esista una dimora e che il soggetto riesca a trovare accoglienza al suo interno, ritirandosi dal mondo delle cose, prendendo le distanze da quell'ambiente anonimo e indeterminato che Lévinas definisce con il termine «élémental» ${ }^{47}$.

Nella segretezza isolata della sua dimora, il soggetto potrà godere passivamente della pura qualità senza sostanza, sperimentandone, tuttavia, anche l'insicurezza. Questa forma della precarietà determina una violenta lacerazione nel profondo del soggetto, aprendone la coscienza alla rivelazione della trascendenza. Le cose, in sé considerate, si trovano in uno stato di ovvia e necessaria acquiescenza - dato che non possono in alcun modo resistere alla conquista. Tuttavia, altri potenziali possessori sono in grado di questionare la legittimità del nostro possesso, perché «di fronte al volto nudo e indigente dell'altro non è possibile un tranquillo possesso del mondo». La rappresentazione delle cose - possibile solo a partire dalla separazione consentita dall'intimità della casa - diviene quindi in grado di esplicare il suo senso solo in virtù del riferimento ad Altri; al contempo, il rapporto con Altri può effettivamente realizzarsi solo attraverso l'offerta di ospitalità ${ }^{48}$.

Le riflessioni sin qui esposte contribuiscono dunque alla genesi di una nuova etica, incentrata sul riconoscimento del volto altrui (visage). Grazie ad essa l'idea di infinito può finalmente «prendere corpo» attraverso l'espressione del viso altrui. A differenza di Heidegger, Lévinas ritiene che l'esperienza del volto altrui non possa essere considerata alla stregua di uno svelamento, come se si trattasse di riportare l'Altro alla «luce» di una comprensione ad esso antecedente, si tratterebbe bensì di una «rivelazione» che consente l'e-

\footnotetext{
46 Totalità e Infinito, cit., 83

$47 \ll$ Raccogliendosi nell'intimità della casa, l'uomo sospende il rapporto di godimento immediato con gli elementi, cessa di esservi semplicemente immerso e può così farne delle «cose», prendendone possesso con il lavoro e fissandoli come sostanze durevoli nella rappresentazione» (FerretTi, G., La filosofia di Levinas, cit.,p. 153).

48 Ferretti, G., La filosofia di Emmanuel Levinas, pp. 154-156.
} 
sperienza assoluta dell'alterità ${ }^{49}$. L'espressione del volto di Altri consentirebbe dunque al soggetto di esperire in maniera «pura» ed «originaria» quella «cosa in sé» che è Altri in quanto Altri. Tutto ciò è possibile perché il volto di Altri è nudo, irrompe come «estraneo» nel mio mondo provocandomi in ragione del suo essere «indigente».

La nudità del volto non si impone al soggetto in ragione di una forza più grande, ma grazie all'infinito della trascendenza etica che annichilisce ogni dimensione di potere: la relazione etica è strutturalmente «asimmetrica», prescinde da qualsiasi forma di reciprocità, essa determina una «curvatura dello spazio intersoggettivo» che situa Altri più in alto di Medesimo. Tutto ciò sta a significare che l'etica - intesa come quella relazione metafisica che si instaura tra Medesimo e l'Altro - possiede un primato sull'ontologia - intesa come lettura di ogni rapporto interpersonale operata sullo sfondo anonimo dell'essere.

Appare dunque evidente la differenza che separa il pensiero levinasiano dalle riflessioni kantiane. Mentre Kant considerava l'ospitalità sotto una luce essenzialmente giuridica e politica, declinandone i tratti fondamentali nel vocabolario delle regole e delle condizioni, per Lévinas l'ospitalità è una condizione originaria ed incondizionata, esattamente come originaria ed incondizionata è l'apertura che orienta la coscienza verso Altri ${ }^{50}$. Sotto un certo punto di vista, la speculazione filosofica levinasiana approfondisce e rivoluziona l'imperativo categorico kantiano, nella misura in cui considera violenta ogni azione che il soggetto compie come se fosse solo e l'universo non avesse altro compito che quello di riceverne (di subirne) l'azione ${ }^{51}$.

49 Come è stato giustamente notato, in tal modo «il filosofo accentua il versante non teoretico della relazione tra lo stesso e l'altro, essenzializzando la dimensione etica: il volto evidenzia infatti una comandamento che investe la coscienza, impedendone una identificazione trionfante di sé con Sè, dal momento che l'unico modo di rifiutare l'epifania dell'Altro nel volto di altri sarebbe l'omicidio, la distruzione dell'umano e la fine della morale» (BREZZI, F., «Reinterrogando Levinas», in Emmanuel Levinas. Prophetic Insipiration and Philosophy, cit., p. 269).

50 Questa differenza non sfugge a DERrIDA, J. (Addio a Emmanuel Lévinas, pp. 112-113). Sul punto, si veda altresì la critica di ANDRONICO, A., La disfuzione del sistema. Invertire, inventare ed ospitare, Giuffrè, Milano, 2006, p. 75.

51 «Nel pensiero di Lévinas, l'imperativo categorico kantiano, che chiede di agire come se il principio della nostra azione dovesse valere universalmente, subisce un approfondimento decisivo, discostandosi dall'ottimismo illuministico che lo contraddistingueva [...] si avverte in questa contestazione, nonostante sia razionalmente e rigorosamente dedotta, l'ammonimento nei confronti di una presunzione rischiosa, quasi una versione tutta ebraica del concetto greco di hybris» (TARTER, S., Evento e ospitalità, cit., p. 76). 
La qualità rivoluzionaria e dirompente della filosofia di Lévinas diviene particolarmente evidente se teniamo a mente che all'interno del contesto culturale moderno, la libertà assume essenzialmente il significato di autonomia, mentre ogni forma di eteronomia è giudicata come una forma di schiavitù. Come diretta conseguenza, all'idea di conflitto interpersonale è stato riservato un ruolo fondamentale ed imprescindibile, incardinando, sotto il segno della dicotomia schmittiana amico-nemico, non solo le modalità attuative della prassi politica, ma l'intero processo di identificazione antropogenico ${ }^{52}$. Dal canto suo, il filosofo di Totalità e infinito ha ritenuto di dover incentrare la propria concezione dell'alterità sulla precedenza dell'altro, ribaltando il rapporto di antecedenza logica tra libertà e responsabilità ${ }^{53}$.

Egli ha inteso in tal modo tracciare la strada verso un «umanesimo altro», in cui «il soggetto non è l'Ego soddisfatto, egoista e solipsista, presente in gran parte della filosofia occidentale, ma il soggetto ospite, responsabile e vulnerabile, il soggetto ostaggio ${ }^{54}$, considerando che solo grazie a questo stravolgimento sarebbe stato possibile «mettere in mora la nozione di affrontamento e contestare il primato del politico» che da essa deriva ${ }^{55}$.

A tal riguardo, è stato correttamente fatto notare che, nella filosofia levinassiana, la «insistente presenza del tema dell'ospitalità non diventa quasi mai problema politico», ed è stato conseguentemente rimproverato all'Autore di non aver mai compiuto «il passo ulteriore per designare un diritto di quella e quindi una prassi politica», come possiamo invece ritrovare, «pur con tutti i limiti del cosmopolitismo settecentesco», in $\mathrm{Kant}^{56}$. Si tratta di una critica condivisibile, ma solo nella misura in cui si rifiuti di porre il pensiero di Lév-

52 Per questo motivo, nella concezione moderna «il politico che, con i suoi concetti di amico e nemico, getta luce sulla contrapposizione più intensa ed estrema di tutte, e che colora di sé tutti gli altri settori della vita, il morale, l'estetico e l'economico, è, come è stato detto» non solo possibile, ma anche reale e necessario (LISSA, G., Dal primato della politica al primato dell'etica, cit., p. 157).

53 «Non c'è dubbio che il pensiero di Emmanuel Levinas sia stato nel Novecento, quello che maggiormente ha contribuito a mettere in crisi questo paradigma a tutt'oggi, per molti versi, imperante. La sua filosofia dell'alterità, incentrata sulla precedenza dell'altro, non solo ha inferto un colpo mortale all'istanza del Soggetto, ma, affermando la priorità della responsabilità sulla libertà, ha operato una vera e propria rivoluzione copernicana, di cui ancora stentiamo a vedere gli effetti» (RESTA, C., L'Estraneo, cit., p. 79).

54 BrezZI, F., Reinterrogando Levinas, in Emmanuel Levinas. Prophetic Insipiration and Philosophy, cit., p. 261.

55 Cfr. Lissa, G., Dal primato della politica al primato dell'etica, cit., p. 168.

56 In tal senso, REZZI, F., Reinterrogando Levinas, cit., p. 271. 
inas al di là del politico, ovvero di accettarne la dimensione necessariamente ultrapolitica. Con ciò non intendo dire che l'etica levinassiana è consapevolmente utopica e non richiede dunque di essere realizzata, ma che porre questa etica del volto e della passività a fondamento di una speculazione filosofica suppone lo sfondamento dell'orizzonte concettuale entro cui la prassi politica è nata e si è sviluppata sino ad oggi ${ }^{57}$, implicando l'avvento di una teoria della comunità che sappia «emanciparsi dall'aderenza all'essere, dall'appartenenza sempre sospetta ad una tradizione, a comuni radici, facendo segno verso quelle rotture che nella storia continuamente la interrompono provenendo da un al di là della politica e da un al di là della storia» ${ }^{58}$.

\section{IL DOVERE DI ESSERE OSPITALI}

Esiste una possibilità di interpretare la libertà di cui gode l'essere umano a prescindere dal pregiudizio filosofico secondo il quale egli rappresenta lo «sguardo primo» che si apre sul mondo, decidendo sovranamente come agire? ${ }^{59}$ La filosofia lévinasiana ha mostrato che «l'altro taglia l'io senza sosta», «lo percorre già da sempre», dovremmo dunque considerare l'alterità alla stregua di un dato originale ed originario ${ }^{60}$. Perché, è stato correttamente affermato, la relazione con gli altri esseri umani rappresenta l'evento, nella sua forma più pura e radicale: solo tramite di essa possiamo apprezzare lo scorrere del tempo ed evitare, in tal modo, la putrefazione solipsitica che

57 Si tratta di una nota caratteristica del pensiero levinasiano sulla quale ha giustamente insistito DerridA, J., Addio a Emmanuel Levinas, cit., p. 148. Al riguardo, Alberto Andronico giustamente annota: «Essendo fenomenologicamente legata all'intenzionalità della coscienza di un soggetto che si trova ad essere già da sempre ricevuto nella sua propria casa, la pace non sarebbe infatti pensabile secondo le tradizionali distinzioni tra politico e non-politico. Non sarebbe insomma né un concetto semplicemente politico, né un concetto, altrettanto semplicemente, estraneo alla politica, ma eccederebbe il politico dal suo interno» (La disfuzione del sistema, cit., p. 76.)

58 In tal modo, SANDRO TARTER ritiene che «anche l'obiezione, rivolta a Lévinas da più parti, di formulare una concezione che non consente la deduzione di una politica o un indirizzo per una prassi concreta troverebbe [...] finalmente una risposta» (evento e ospitaità, cit., 91)

59 Riprendo questo lacerante interrogativo da TARTER, S. (evento e ospitalità, cit. 76 e ss.). A tal riguardo, l'autore afferma che «la contestazione della libertà come primum è forse una delle mosse più sconcertanti di Lèvinas, che conferisce al suo pensiero una forte componente di inattualità. La libertà non viene mai celebrata come un valore di per sé, anzi, se pensata a prescindere dalla giustizia, finisce per rivelarsi come la matrice stessa della violenza».

60 «Chi può mai dire io senza aver già da sempre ammesso in esso la presenza dirompente del $t u$ ? » (Coccolini, G., Ospite Ostaggio Nemico, cit., 14). 
affligge Medesimo ${ }^{61}$. Vivere in relazione significa sperimentare una valorosa lacerazione coscienziale, implica che la soggettività non venga più considerata alla stregua di una forma della sovranità, quasi che essa stessa fosse il centro del mondo, venendo al contrario costruita ed interpretata grazie ad una diversa chiave ermeneutica, rappresentata dalla dipendenza e dallo svuotamento $^{62}$.

Dal punto di vista strettamente giusfilosofico, queste riflessioni ci possono aiutare a configurare un dovere fondamentale di essere ospitali. Sino a quando concepiremo la comunità come qualcosa che esiste solo se i suoi componenti sono legati insieme da un elemento che li accomuna, e quindi da un qualcosa che essi già possiedono uti singuli, non saremo in grado di bandire dal suo interno la violenza e l'esclusione ${ }^{63}$. Perché la logica integrativa-escludente della politica ha da sempre fatto perno sull'ideale e comune proprietà di un bene che, appartenendo ai membri del gruppo, dovrebbe essere da questi gelosamente custodito e difeso - come se la universale condivisione di quello stesso bene comune ne potesse determinare la dispersione, decretando la morte del gruppo politico per consunzione ${ }^{64}$.

61 Non tutto ciò che accade può essere definito, per ciò stesso, eventuale, ma solo ciò che e-viene nella sua assoluta e irriducibile singolarità, sfondando il nostro orizzonte di attesa e consentendoci l'esperienza esorbitante di una eterogeneità sconvolgente, perché imprevista e quindi assolutamente intangibile. In tal senso, l'a-venire dell'altro rappresenta la «precipitazione di una singolarità assoluta» che si lega «necessariamente alla forma dell'istante, nell'immanenza e nell'urgenza» (DERRIDA, J., Spettri di Marx. Stato del debito, lavoro del lutto e nuova internazionale, Milano 1994, 60; per un commento Resta, C., L'evento dell'altro: etica e politica in Facques Derrida, Bollati Boringhieri, Torino, 2003, 32 e ss).

62 Coccolini, G., Ospite Ostaggio Nemico, cit., 14.

63 È stato giustamente notato che con l'espressione «bene comune» non dobbiamo fare riferimento «a una generalidad que elimina particularidades, no es un bien que anule las subjetividades, tampoco equivale al concepto de interés generale consagrado por el liberalismo anglosajón y que incide en el aspecto individual y subjetivo» (DíAZ DE TERÁN, $M^{a}$ C., Multiculturalismo, bioética y derecho, in Á. Aparisi Miralles e $M^{\mathrm{a}}$ Cruz Díaz de Terán [a cura di], Pluralismo culutural y democracia, Thomson Reuters, Navarra 2009, 209).

64 Per questo motivo «l'ontologia regionale della politica racchiude in sé un'ambivalenza insopprimibile» in base alla quale «tanto più il rapporto politico è unitario e integrativo, e perciò pacificante all'interno, tanto più è separante verso l'esterno». Per di più, si tratta di un'ambivalenza insuperabile, perché il bene comune deve «essere effettivamente partecipato o sentito come effettivamente partecipabile; ma per ciò stesso è cio che più separa dagli stranieri, i quali non vi hanno parte alcuna; va quindi difeso contro di essi quale elemento determinante dell'identità politica comune» (COTTA, S., Il diritto nell'esistenza. Linee di ontofenomenologia giuridica, Giuffrè, Milano 1992, 115-119). 
Sino a quando continueremo a pensare la comunità in questi termini, noi saremo destinati a fallire clamorosamente nella ricerca del senso ad essa sotteso. La comunità non nasce infatti quando molti uomini si uniscono sotto l'egida di un elemento terzo che tutti già dominano e possiedono $u t i$ singuli, ma è tale quando essi decidono di legarsi reciprocamente nel nome di un elemento che non appartiene loro e del quale non possono appropriarsi, né uti singuli né come gruppo. Per questo motivo non possono essere condivise le teorie comunitarie contemporanee, che, nel tentativo di contrastare il compimento post-moderno del nichilismo, propongono una concezione della comunità come pienezza $a^{65}$. In un certo qual modo, i communitarians risultano incapaci di uscire dalla prospettiva individualistica, continuando a pensare la società in termini antropomorfici e hobbesiani, come se fosse un individuo che può collaborare con (o contrapporsi a) altri individui ${ }^{66}$.

Possiamo invece costruire una comunità aperta e ospitale accettando di entrare nella logica del munus reciproco: a patto di saper tradire la nostra attitudine immunitaria, ovvero, la nostra spontanea tendenza a reagire nei confronti di ogni agente antigeno, scatenando contro di esso quegli anticorpi che, nelle intenzioni, ci consentirebbero di restare fedeli alla nostra tradizione ${ }^{67}$. Se vogliamo fondare una comunità dell'accoglienza dobbiamo

65 Insomma, ogni teoria della comunità che prenda le mosse dal tema della appropriazione, comporta, paradossalmente, la definitiva scomparsa della possibilità stessa di creare una comunità. In termini heideggeriani: la cosa è annichilita quando non se ne coglie sino in fondo quel carattere essenziale che corrisponde, esattamente, con il vuoto che la costituisce internamente. Per questo motivo, nella conferenza Das Ding, Martin Heidegger richiamò alla mente del suo uditorio alcune immagini parecchio significative: l'albero; il ponte; la soglia... si tratta di figure della relazione che unisce (cielo e terra, le due sponde del fiume, l'interno della casa con l'esterno). Allo stesso modo, la brocca, esempio principe all'interno di quel discorso, esiste attorno ad un vuoto, non perché essa sia questo vuoto, ma perché è grazie ad esso che può svolgere la propria funzione che è, esattamente, quella di offrire il suo contenuto, creando in tal modo una relazione, mettendo in comune.

66 Per quanto riguarda le teorie dei liberals e dei communitarians, si veda Amato MangIAMELI, A. C., «Desiderai essere un cittadino». Oltre il retaggio simbolico della moderna sovranità, Giappichelli, Torino 1996, 17-67.

67 «Lidea di communitas - e, prima ancora, di munus, da cui questa deriva - va in un senso radicalmente contrario a tale irrefrenabile spinta all'interiorizzazione, ma si dovrebbe dire «internamento», di tipo immunitario. Essa rimanda, al contrario, a una esteriorizzazione dell'esistenza; $\mathrm{o}$, ancor meglio, a un'interpretazione della stessa esistenza come esteriorità, esperienza, estasi, nel senso radicale di queste espressioni: come fuoriuscita del soggetto da se stesso o come sua apertura originaria all'alterità che lo costituisce fin dall'inizio nella forma di un «essere con»o di un «con-essere» (cfr. EsPosiTo, R., Communitas, cit.). 
provare a partire da ciò che manca, dobbiamo saper prendere le mosse da quel di meno che potrebbe legare gli esseri umani nel nome della loro strutturale debolezza, della loro originaria fallacia. Prendendo le mosse da questa difettività - che la tradizione veterotestamentaria definiva con il nome di nefes - è possibile immaginare una comunità capace di accogliere, senza per ciò solo correre il rischio di normalizzare o inglobare, perché il comune denominatore, la base in ragione della quale essa esiste, è che essa non domina e non possiede. In tal modo, lo straniero non sarebbe più considerato alla stregua di un pericolo che, venendo dall'esterno, infetta, superandoli, i margini identitari, ma rappresenterebbe al contrario la conferma dei presupposti su cui l'identità collettiva è stata pensata e costruita.

Questa è la legge dell'ospitalità alla quale siamo chiamati, una regola che non sempre è tenuta nella giusta considerazione che spesso viene tradita dalle leggi sull'ospitalità ${ }^{68}$, ovvero da quel sistema di scambi e di reciproche concessioni che costituisce il vocabolario dell'economia, prima ancora che del diritto. Per questo motivo, possiamo ben dire che la comunità non è pienezza né appropriazione. La comunità non colma le lacune del soggetto, non attribuisce al singolo qualcosa che egli, da solo, non potrebbe ottenere, al contrario, essa impone lo svuotamento, la mancanza, la lacerazione ${ }^{69}$.

Solo accettando questi presupposti potremmo evitare è che la sfera pubblica diventi il luogo in cui gli uomini entrano in relazione nella forma della loro dissociazione, ovvero, evitare che la logica lacerante e violenta della immunità prevalga su quella della comunità. In tutto questo discorso il tema della giustizia gioca un ruolo di primaria importanza che merita di essere ben messo in evidenza. Prima di tutto, perché, considerando la questione dal punto di vista strettamente metafisico, possiamo affermare che l'ospitalità rappresenta la

68 Per un commento, BrezZI, F., Reinterrogando Lévinas, in Emmanuel Lévinas, cit., 217-229.

69 E' in questo senso che Roberto Esposito scrive che «la comunità non è il luogo della contrapposizione, ma quello della sovrapposizione, tra cosa e niente»; è in questo senso che possiamo elaborare un parallelismo tra comunità e nichilismo andando oltre una apparente e netta contrapposizione, per sottolineare il punto in cui questi due concetti, che non possono essere né situati sullo stesso piano, né tantomeno, immaginati sulla stessa identica traiettoria, si incrociano. La comunità nasce nel momento in cui il soggetto concede agli altri il suo munus (un dono che, propriamente, viene appunto concesso, fatto e non ricevuto) proiettando la propria identità verso l'esterno, la comunità prende vita nel momento in cui l'individuo (dis)perde una parte della propria soggettività, facendone dono agli altri, essa è quindi il risultato di un atto di liberalità che ba il sapore della liberazione (Termini della politica. Comunità, Immunità, Biopolitica, Einaudi, Milano-Udine, 2008-2009.) 
pre-condizione della giustizia, ovvero che non esiste giustizia senza ospitalità. Perché l'idea stessa di giustizia implica che il soggetto sia in grado di farsi da parte, di scavare dentro di sé e trovare un posto per le legittime pretese altrui, di creare uno spazio affinché l'altro abbia ciò che gli spetta. In tal senso, il dovere di essere ospitali si rivela il primo e fondamentale dovere dell'uomo.

A questa prima riflessione, di stampo chiaramente e metaforicamente allusivo, ne dobbiamo aggiungere un'altra, forse più e urgente nell'attuale scenario geopolitico e che tuttavia consegue direttamente dalla prima: non ha alcun senso proclamare da un lato l'universalità dei diritti dell'uomo e dall'altro rifiutare accoglienza e ospitalità agli stranieri che si trovino in pericolo di vita. Sul punto è bene essere chiari. Questo saggio non intende in alcun modo strumentalizzare le riflessioni freudiane, la sociologia simmeliana o la filosofia di Lévinas per caldeggiare la totale scomparsa dei confini statuali. Al contrario, muove dal presupposto che il confine è un elemento identitario di fondamentale importanza e che un suo totale superamento non sarebbe non solo possibile, ma nemmeno auspicabile. Ciò che ho inteso invece fare è stato mostrare l'importanza dell'accoglienza e dell'apertura nei confronti dello straniero in quanto soggetto debole. Volendo quindi compiere quel passaggio dalla speculazione teoretica alla filosofia del diritto che molti imputano di non aver mai compiuto allo stesso Lévinas, a me sembra evidente che il rispetto dei diritti dell'uomo ci imponga di accogliere senza se e senza ma chiunque stia fuggendo da una guerra, da una persecuzione o dalla carestia. In tutti questi casi, il dovere di essere ospitali si rivela un dovere fondamentale dal quale non possiamo davvero prescindere, se vogliamo dotare di coerenza e di senso il nostro fattivo impegno per il riconoscimento e per l'effettività dei diritti dell'uomo.

\section{BIBLIOGRAFIA}

Amato Mangiameli, A. C., «Desiderai essere un cittadino». Oltre il retaggio simbolico della moderna sovranità, Giappichelli, Torino, 1996.

ANDronico, A., La disfuzione del sistema. Invertire, inventare ed ospitare, Giuffrè, Milano, 2006.

Benveniste, E., Vocabolario delle istituzioni indoeuropee. Economia, parentela, società, Einaudi, Torino, 2001.

BilotTa, B.M., L'ospite poco ospite, in B. M. Bilotta e F. A. Cappelletti (a cura di), Il diritto d'asilo, Cedam, Padova, 2006.

Cacciari, M., Geofilosofia dell'Europa, Adelphi, Milano, 1994. 
Carotenuto, A., Il fascino discreto dell'orrore, Bompiani, Bologna 2002.

Coccolini, G., Ospite Ostaggio Nemico, Pardes, Bologna, 2004.

Conte, A. G., Elend: il linguaggio dell'alterità, in AA. VV., in R. Astorri e F. A. Cappelletti (a cura di) Lo Straniero e l'ospite. Diritto Società Cultura, Giapichelli, Torino, 2002.

CotтA, S., Il diritto nell'esistenza. Linee di ontofenomenologia giuridica, Giuffrè, Milano 1992.

Curi, U., Straniero, Raffaello Cortina, Milano, 2010.

Dal LAGO, A., Non-persone. L'esclusione dei migranti in una società globale, Feltrinelli, Milano, 2004.

Derrida, J., Spettri di Marx. Stato del debito, lavoro del lutto e nuova internazionale, Raffaello Cortina, Milano, 1994.

- Addio a Emmanuel Lévinas, Jaca Book, Milano, 1998.

- Sull'ospitalità, Raffaello Cortina, Milano, 2000.

- Scrittura e differenza, Einaudi, Torino, 2004.

Di SANTE, C., Lo straniero nella bibbia. Saggio sull'ospitalità, San Paolo, Troina (EN), 2002.

DíAz DE TERÁN, $M^{\mathrm{a}}$ C., Multiculturalismo, bioética y derecho, in Á. Aparisi Miralles e $M^{a}$ Cruz Díaz de Terán (a cura di), Pluralismo culutural y democracia, Thomson Reuters, Navarra, 2009.

Dussel, E., Filosofia della liberazione, Queriniana, Brescia, 1992.

Esposito, R., Communitas. Origine e destino della comunità, Einaudi, Torino, 2006.

- Termini della politica. Comunità, Immunità, Biopolitica, Einaudi, Milano-Udine 2008-2009.

Ferretti, G., La filosofia di Levinas: alterità e trascendenza, Rosenberg \& Sellier, Torino, 1996.

Freud, S., Opere, Parte II, Bollati Boringhieri, Torino, 2006.

Gentili, D., Topografie politiche. Spazio urbano, cittadinanza, confini in Walter Benjamin e Facques Derrida, Quodlibet, Macerata, 2009.

Jonas, H., Lo gnosticismo, SEI, Torino, 1991.

LÉVINAs, E., L'ontologia è fondamentale?, in Tra di noi. Saggi sul pensare-all'altro, Jaca Book, Milano, 2000.

- Il Tempo e l'Altro, Il Nuovo Melangolo, Genova, 2005.

- Alterità e trascendenza, Il Nuovo Melangolo, Genova, 2006.

LISSA, G., Dal primato della politica al primato dell'etica, in I. Kajon, E. Baccarini, F. Brezzi e J. Hansel (a cura di), Emmanuel Levinas. Prophetic Inspiration and Philosophy, Giuntina, Firenze, 2008.

Perrone, L., Da straniero a clandestino. Lo straniero nel pensiero sociologico occidentale, Liguori, Napoli, 2005.

Resta, C., L'evento dell'altro: etica e politica in Facques Derrida, Bollati Boringhieri, Torino, 2003.

- L'Estraneo. Ostilità e ospitalità nel pensiero del Novecento, Il Nuovo Melangolo, Genova, 2008. 
RovatTi, P.A., Intorno a Lévinas, Unicopli, Milano, 1987.

Salvarezza, F., Emmanuel Lévinas, Mondadori, Milano, 2003.

Simmel, G., Sociologia, Meltemi, Torino, 1998.

STRAUSS, C. L. Razza e storia e altri studi di antropologia, Einaudi, Torino, 1967.

TABBOnI, S., Lo straniero e l'altro, Liguori, Napoli, 2006.

TARTER, S., evento e ospitalità. Levinas, Derrida e la questione straniera, Cittadella, Assisi, 2004.

Todorov, T., La conquista dell'America e il problema dell'altro, Einaudi, Torino, 2014.

VITALE, E., Ius migrandi, figure di erranti al di qual della cosmopoli, Bollati Boringhieri, Torino, 2004. 
- 000 